\title{
Structural Layers Origin Of The Blue Colour Reflections On The Wings Of The Junonia Orithya Madagascarensis
}

\author{
Issaka Ouedraogo, PhD \\ Emmanuel Nanema, PhD \\ Research Institute of Applied Science and Technology, \\ Ouagadougou, Burkina Faso \\ Bintou Ouedraogo, PhD \\ Alioune Ouedraogo, Prof. \\ Ouagadougou University- UFR-SEA Ouagadougou, Burkina Faso \\ Priscilla Simonis, PhD \\ Research Center in Physics of Matter and Radiation. \\ Namur University, Belgium \\ Belkacem Zeghmati, Prof. \\ Laboratory of Mathematics and Physics Systems, \\ University of Perpignan, France
}

doi: 10.19044/esj.2016.v12n24p147 URL:http://dx.doi.org/10.19044/esj.2016.v12n24p147

\begin{abstract}
This article is devoted to the study of the structural layers origin of the blue reflections on the scales of the wings of the Junonia orithya madagascarensis, a butterfly of the Nymphalidae species. We proceed by spectrophotometry and scanning electron microscope (SEM) characterization of these layers to explain the origin of the blue color of the wings. We also made numerical calculations to simulate the structural nature of these layers which help to support the experimental results. Indeed, from the measurements using the spectrophotometer, a main peak of reflection was obtained at $483 \mathrm{~nm}$. From our calculations results we get a $515 \mathrm{~nm}$ for the dominant length in reflection and $510 \mathrm{~nm}$ for the numerical reproduction of the reflectance spectrum, respectively. These results confirm that the multilayer structure is at the origin of the blue colour of the dorsal scales of the wings of the Junonia. A very thin membrane is responsible for it. This membrane diffuses sunlight at its upper and inner surface. Therefore, it is called structural coloration. It is possible to consider artificial reproduction for the multilayer through a process of deposits in order to manufacture materials at nanometer scale with selective reflection.
\end{abstract}


Keywords: Reflectance, blue color, structural coloration

\section{Introduction}

In the wide variety of living organisms in the natural world, we encounter insects, such as butterflies, whose wings have structure layers of colors called photonic, which control the propagation of light within them (L. Biro and al, 2007; P. Vukusic and al, 2003; M. Land, 1972). These structures allow us to differentiate pigment or structural origin of these colorations. In general, the pigment coloration is more common in the nature of living organisms. Specifically, it is the geometry of the chemical that creates the impression of colour. On the contrary, structural colour is due to a physical phenomenon, typically by diffraction or interference (Y. Nekrutenko and al, 1965; J.P. Vigneron and al, 2006; Zs. Balint and al, 2005). In the realm of insects such as butterflies, colour is fundamental to the survival of the species. In fact, they use it to move, recognize each other and avoid predators. Figure (1) shows a butterfly, Junonia orithya madagascenris, which lives in tropical climates and whose dorsal wings have a blue colour. This species was collected in 2008 in the botanical garden, preserved for more than 5 years at the Natural History Museum of the National Center of Scientific and Technological Research of Burkina Faso. The butterfly Junonia has never been studied to our knowledge for the colour of its wings. During our study, we show the origin of the blue coloration of the wings of the butterfly Junonia and the structure that is responsible. For this, we use an experimental approach, the spectrophotometer, the Scanning Electron Microscope (SEM), and finally a numerical method in order to compare the different results.

\section{Junonia orithya madagascariensis}

Butterfly Junonia orithya madagascariensis is a butterfly of the family Nymphalidae with about 6,000 species around the world. (S. Berthier, 2007) Most often, they are encountered in the borders of the Indian Ocean and in tropical climates in West Africa. They feed on young leaves of plants. The shape and size of the wings are used to differentiate the female and the male within the same species. Generally, the female is larger with a few centimeters. The wings are made of a double membrane, each one having developed independently and joined during the final constitution of the wings. Thus, the ventral and dorsal sides are completely covered with feathers called scales. The wings have the shape of two triangles with rounded corners. The ratio between the size $(35-45 \mathrm{~cm})$ of the wings and that of the body (the weight $0.5 \mathrm{~g}$ ) is an important parameter in the life of the butterfly. The wings are characterized on the edge by a row of several 
eyespots of orange colour with black in the centre, and on the other orange eyespots with white in the centre. At the dorsal side of Junonia towards the head, there is a black surface dotted with sky blue grain. The dorsal part towards the tail of Junonia has a blue colour surface. The blue coloration of the scales of the dorsal surface of the Junonia is a subject of special attention in this study.

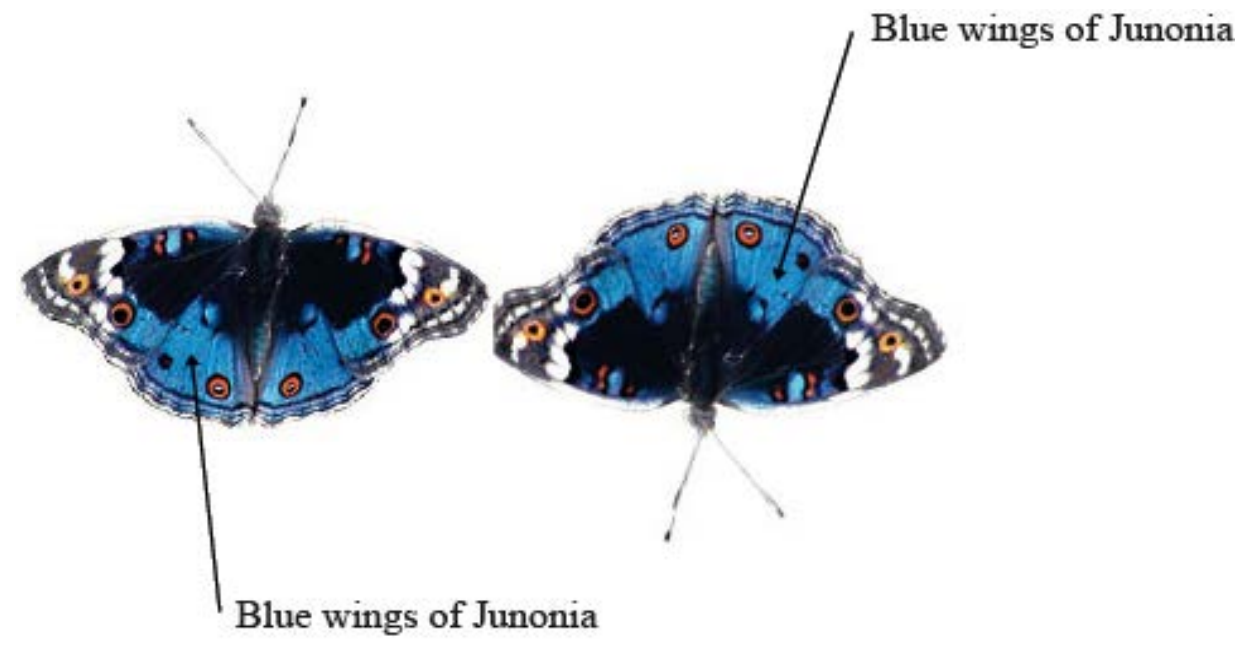

Fig.(1).Butterfly Junonia orithya madagascariensis male

\section{Spectroscopic measurements}

A piece of wing of dorsal side of Junonia is collected and analyzed in a spectrophotometer-Avaspec-2048 optical fiber. The spectral range of this unit extends from $250 \mathrm{~nm}$ to $900 \mathrm{~nm}$. Its main advantage is its ease of use and speed of acquisition of the spectrum. Indeed, the entire spectrum, reflectance or transmission is achieved instantly, with a resolution of about 5 $\mathrm{nm}$. In the case of Avaspec-2048, the reference is a white diffuser and the material of reflectance spectrum is greater than $98 \%$ in the visible. The measurements are performed on samples of $0.5 \times 0.5 \mathrm{~cm}$ that are cut in the wings of the dorsal surface of the male specimen. We measure the sample spectrum for incidence angles $\theta$ from $0^{\circ}$ to $60^{\circ}$ in $15^{\circ}$, used to find the reflected or transmitted intensity. We Present results for $\theta$ equal to $30^{\circ}$. Other angles do doesn't provide additional information. Figure (2) shows the spectrum for a piece of dorsal scales of the wings of Junonia. There is a main peak at $483 \mathrm{~nm}$. The main peak is the blue color, compared to the chromaticity diagram established by the International Commission on Illumination (ICI) (ICI, Cambridge University Press, 1931). We also, notice between $400 \mathrm{~nm}$ and $500 \mathrm{~nm}$, a harmonic spectrum with very low amplitude at $450 \mathrm{~nm}$. 


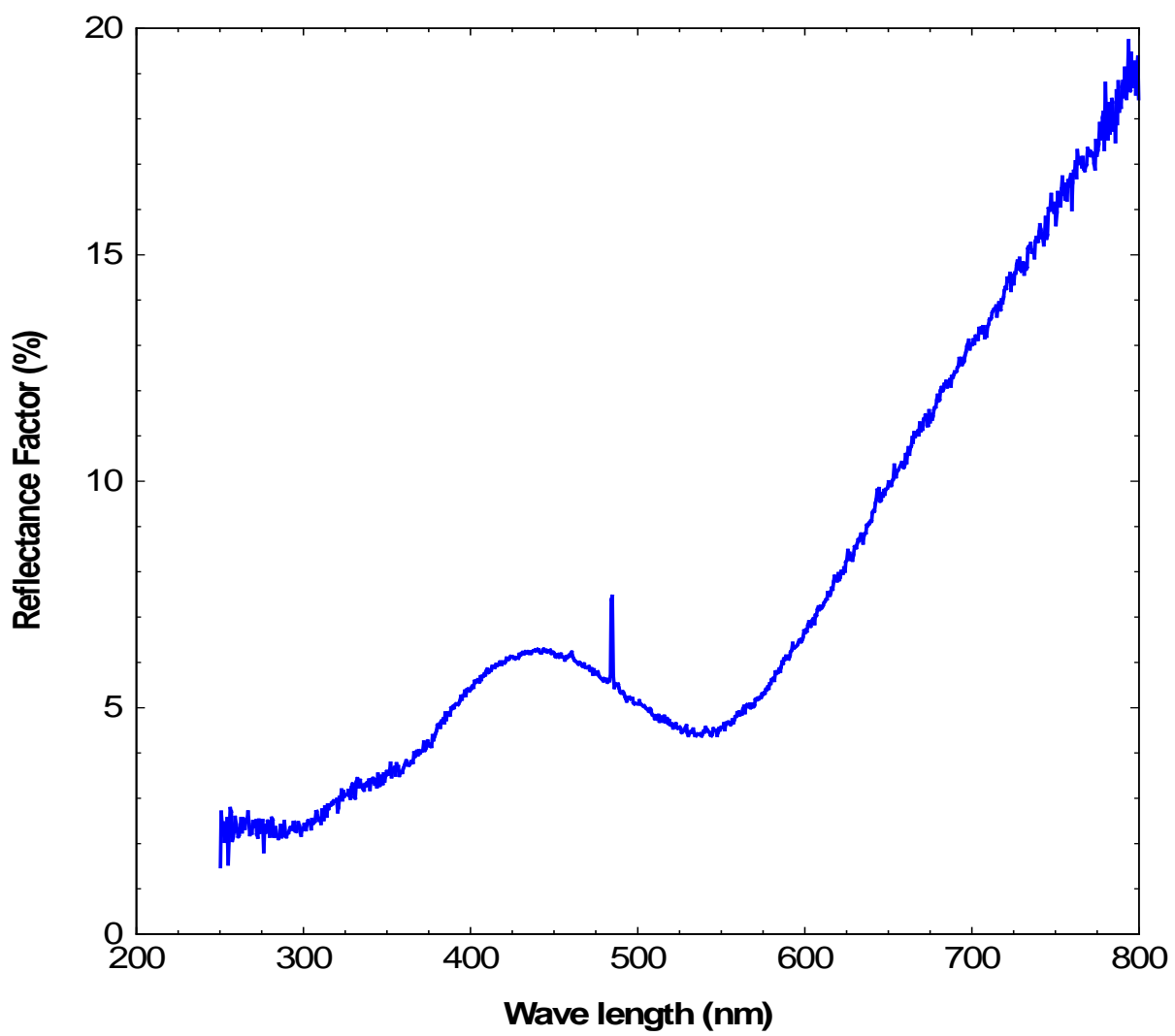

Fig.(2): Junonia reflectance spectrum measured at an incident angle $\theta=30^{\circ}$

\section{Microscopic measures}

Scanning Electron Microscopy SEM (Joel-5510) allows a resolution of about $10 \mathrm{~nm}$. This resolution allows us to both observe and measure the structures that cause the coloring phenomena. All equipment is maintained under vacuum, which avoids the diffusion of the beam of electrons.

During the review with SEM, samples of $0.5 \times 0.5 \mathrm{~cm}$ are subject to electronic load bombarding. If the sample is an insulator, that is to say, if it pushes back the electron beam, as it is in the case of Junonia, we deposit on the specimen a thin layer of gold of approximately $10 \mathrm{~nm}$, to eliminate the negative charges, leading them to the mass of the microscope. After using the spectrophotometer-Avaspec, we go to the SEM for observation at very low scale of a piece of the scales of wings of butterfly Junonia. We note a stack of layers of homogenous and parallel length. These different layers are composed of chitin and chitin-air mixed layers. The number of layers is estimated to be between 7 to 8 , which means there is a multilayer. To better see the structure, we perform a vertical section in liquid nitrogen, to obtain a clean break. Figure (3) shows SEM images of the structure at the broken wall 
(left). The image at right shows the internal structure. Image analysis with SEM shows that the multilayer structure is responsible for the blue colour of the dorsal scales of the wings of butterfly Junonia orithya madagascenris. This is a very thin membrane of approximately $200 \mathrm{~nm}$. It has an interesting property because it diffuses part of the rays to its surface but also inside; the refracted rays are diffused toward the outer surface.

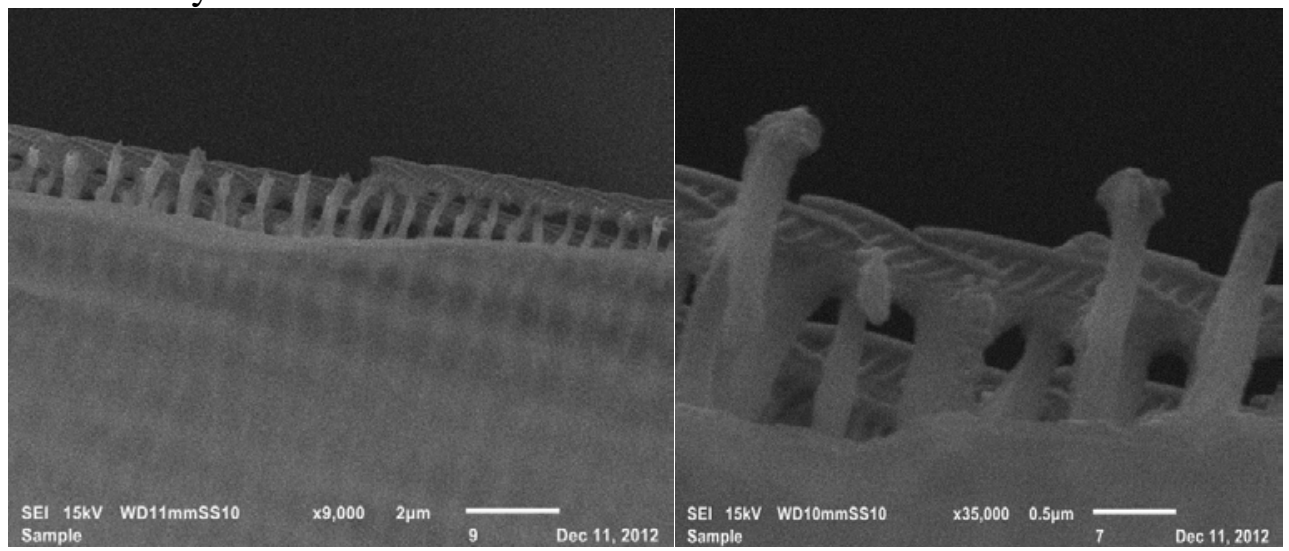

Fig. (3): Observed SEM image of the edge of the break structure (left), and the internal structure (right). Mag= x 45000.

\section{Calculation of the dominant reflected wavelength}

It is possible to determine the dominant wavelength reflected from the multilayer butterfly Junonia, by the following formula (J. Prendry and Mc. Kinnon, 2005; P. Yeh, 2005).

$$
\lambda=\frac{2 \mathrm{a} \sqrt{\overline{\mathrm{n}}^{2}-\sin \theta^{2}}}{\mathrm{~m}}=\frac{2.185 \sqrt{1.48^{2}-\sin ^{2}\left(30^{\circ}\right)}}{1} \simeq 515 \mathrm{~nm}
$$

Note that $\theta=30^{\circ}$ is defined as an angle of incidence relative to the surface of the multilayer. The period is determined by using SEM images, $\mathrm{a}=185 \mathrm{~nm}$. We use the values of thicknesses supplied by the SEM images and the dielectric constants found in the literature for air and chitin. In practice, we doesn't proceed with the refractive indices, but with dielectric constants $(\bar{n}=\bar{\varepsilon}$ is average dielectric constant) $\bar{n}=\sqrt{\bar{\varepsilon}}, \bar{n}=1.48$. Very often in biological systems, ( $m$ is positive integer), $m=1$. The wavelength is estimated at $515 \mathrm{~nm}$, and remains in the area of the blue color. $\lambda$ is in good agreement with the measured spectrum to $483 \mathrm{~nm}$. The calculation of the dominant wavelength in reflection gives a result that is fairly close to the results of measurements. It only allows us to account for the main peak of the reflectance spectra, and beyond this value no wave reflection is possible; it is also called the Bragg mirror. 


\section{Numerical modeling method}

We use the method of transfer matrices to numerical reproduce the reflectance spectrum of the blue wings of the butterfly Junonia (Y. Hoo and al, 2003; J. Lafait and al, 2010; K. Kertesz and al, 2008; L. Biró and al, 2003; J. Zi and al, 2003). The method involves solving Maxwell's equations, which reveals a possible separation of the field components (R. Potyrailo and al, 2007; J. Vigneron and al, 2010; K. Watanabe and, 2005; C.W. Mason, 1927). The first group contains only $E_{x}, H_{y}$ and $H_{z}\left(E_{x}=\right.$ Electric field in (x) axis, $\mathrm{H}_{\mathrm{y}}=$ Magnetic field in (y) axis, $\mathrm{H}_{\mathrm{z}}=$ Magnetic field in (z) axis). And the second involves only $\mathrm{H}_{\mathrm{x}}, \mathrm{E}_{\mathrm{y}}$ and $\mathrm{E}_{\mathrm{z}}\left(\mathrm{H}_{\mathrm{x}}=\right.$ Magnetic field in $(\mathrm{x})$ axis, $\mathrm{E}_{\mathrm{y}}=$ Electric field in (y) axis, $E_{y}=$ Electric field in (z) axis). In the first case, the electric field is reduced to its component $E_{x}$ perpendicular to the plane of incidence $(\theta)$. This is an Electric Transverse Mode (ETM). In the second case, the magnetic field is reduced to its component $\mathrm{H}_{\mathrm{x}}$ that is perpendicular to the plane of incidence $(\theta)$. This is a Magnetic Transverse Mode (MTM). This separation leads to two groups of solutions.

The first

group,

ETM:

$\mathrm{E}_{\mathrm{x}}, \mathrm{H}_{\mathrm{y}}, \mathrm{H}_{\mathrm{z}}$ and $\mathrm{E}_{\mathrm{y}}=\mathrm{E}_{\mathrm{z}}=\mathrm{H}_{\mathrm{x}}=0$

The second group, MTM: $\mathrm{H}_{\mathrm{x}}, \mathrm{E}_{\mathrm{y}}, \mathrm{E}_{\mathrm{z}}=0$ and $\mathrm{H}_{\mathrm{y}}=\mathrm{E}_{\mathrm{z}}=\mathrm{E}_{\mathrm{x}}=0$

Then we can write the wave equations on each of the two groups:

ETM: $\frac{d^{2} E_{x}}{d z^{2}}+\left(\varepsilon(z) \frac{\omega^{2}}{c^{2}}-k_{y}^{2}\right) E_{x}=0$

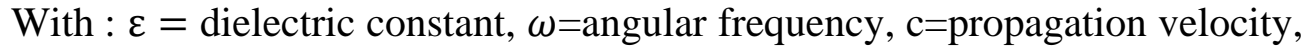
$\mathrm{k}_{\mathrm{y}}=$ component of the wave vector

MTM: $\varepsilon(\mathrm{z}) \frac{\mathrm{d}}{\mathrm{dz}}\left(\frac{1}{\varepsilon(\mathrm{z})} \frac{\mathrm{dH}_{\mathrm{x}}}{\mathrm{dz}}\right)+\left(\varepsilon(\mathrm{z}) \frac{\omega^{2}}{\mathrm{c}^{2}}-\mathrm{k}_{\mathrm{y}}^{2}\right) \mathrm{H}_{\mathrm{x}}=0$

We assume that $\varepsilon(\mathrm{z})$ is constant by piece with matching conditions in $\mathrm{E}_{\mathrm{x}}$ and $\mathrm{H}_{\mathrm{x}}$. We modify the multilayer by introducing a zero thickness and a constant dielectric $\varepsilon_{\text {art }}$ layer between each layer. It is simply a calculation artifice.

\section{Electric Transverse Mode:}

In the ETM, we consider a dielectric constant layer $\varepsilon_{\mathrm{j}}(\mathrm{j}=1$ or 2$)$, flanked by two artificial layers $\varepsilon_{\text {art }}$. Basing on the equation (3), we find the equations describing the interface, and the two artificial layers are the following:

For

the

interface:

$\frac{d^{2} E_{x}}{d z^{2}}+\left(\varepsilon_{j} \frac{\omega^{2}}{c^{2}}-k_{y}^{2}\right) E_{x}=0$

For the two artificial layers

$: \frac{\mathrm{d}^{2} \mathrm{E}_{\mathrm{x}}}{\mathrm{dz}^{2}}+\left(\varepsilon_{\mathrm{art}} \frac{\omega^{2}}{\mathrm{c}^{2}}-\mathrm{k}_{\mathrm{y}}^{2}\right) \mathrm{E}_{\mathrm{x}}=0$

We state: 


$$
\begin{aligned}
& \mathrm{k}_{\mathrm{y}}=\sqrt{\varepsilon_{\mathrm{j}} \frac{\omega^{2}}{\mathrm{c}^{2}}-\mathrm{k}_{\mathrm{y}}^{2}} \\
& \mathrm{k}_{\mathrm{art}}=\sqrt{\varepsilon_{\mathrm{art}} \frac{\omega^{2}}{\mathrm{c}^{2}}-\mathrm{k}_{\mathrm{y}}^{2}}
\end{aligned}
$$

With the following conditions (i represents either the layer $\mathrm{j}$ or the artificial layer):

if $\varepsilon_{i} \frac{\omega^{2}}{c^{2}} \geq k_{y}^{2}$ then $k_{i} \geq 0$

With $\varepsilon_{\mathrm{i}}=$ layer (i) dielectric constant

if $\varepsilon_{i} \frac{\omega^{2}}{c^{2}}<k_{y}^{2}$ then $\operatorname{Im}\left(k_{i}\right)>0$

Where Im=Imaginary part

We note that the second case is not present for the first interface. Indeed, provided that it coincides with the dielectric constant of the incidence medium, we have:

$$
k_{i}=\sqrt{\varepsilon_{\text {art }}} \frac{\omega}{c} \sin \theta \leq \sqrt{\varepsilon_{\text {art }}} \frac{\omega}{c}
$$

For the determined three zones (the layer $\mathrm{j}$ and the two artificial layers surrounding it), we have the following three equations for the electric field along the (x) axis: amplitude is modulated in the direction $(\mathrm{z})$

$$
\begin{gathered}
E_{x}(z)=A_{j} e^{i k_{a r t}}\left(z-z_{j}\right)+B_{j} e^{-i k_{a r t}}\left(z-z_{j}\right) \\
E_{x}(z)=C e^{i k_{j}}\left(z-z_{j}\right)+\operatorname{De}^{-i k_{j}}\left(z-z_{j}\right) \\
E_{x}(z)=A_{j+1} e^{i k_{a r t}}\left(z-z_{j+1}\right)+B_{j+1} e^{-i k_{a r t}}\left(z-z_{j+1}\right)
\end{gathered}
$$

Now we can determine the coefficients $A_{j}$ and $B_{j}$ in terms of $A_{j+1}$ and $\mathrm{B}_{\mathrm{j}+1}$. We have:

$$
\left(\begin{array}{l}
A_{j} \\
B_{j}
\end{array}\right)=T_{j}\left(\begin{array}{l}
A_{j+1} \\
B_{j+1}
\end{array}\right)
$$

$T_{j}$ is the transfer matrix of layer $j$. Using the same process for all layers, we finally obtain the matrix equation of the entire multilayer:

$$
\begin{gathered}
\left(\begin{array}{c}
A_{0} \\
B_{0}
\end{array}\right) \\
=\underbrace{\mathrm{T} 1, \mathrm{~T} 2, \mathrm{~T} 3 \ldots \mathrm{T}_{\mathrm{n}}}_{\mathrm{T}}\left(\begin{array}{l}
\mathrm{A}_{\mathrm{n}+1} \\
\mathrm{~B}_{\mathrm{n}+1}
\end{array}\right)
\end{gathered}
$$

Where $\mathrm{T}$ is the transfer matrix of the multilayer.

\section{The Magnetic Transverse Mode}

We use the same reasoning of the Electric Transverse Mode (ETM), but from equations (Eq.4) and (Eq.10) to obtain matrix Tj. We reiterate to obtain transfer matrix $\mathrm{T}$. 
Knowledge of the transfer matrices of the two polarization modes ETM and MTM are used to calculate the coefficients of reflectance and transmittance. A computer code was developed to calculate the reflectance and the transmittance spectrum of the multilayer. The numerical modeling results by the above method are presented by Figure (4).

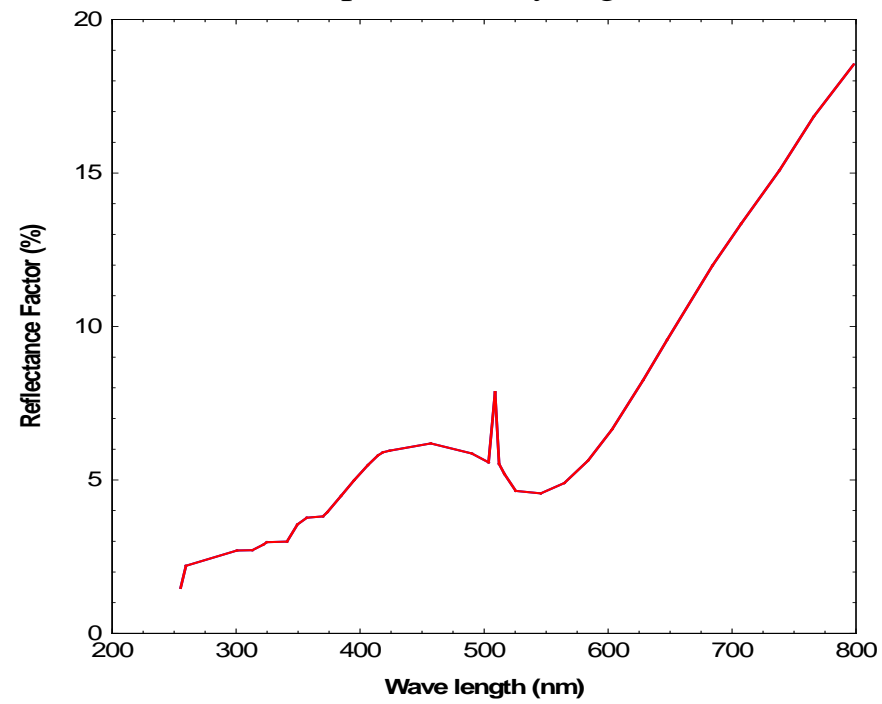

Fig.(4) Junonia reflectance spectrum simulated at an incident angle $\theta=30^{\circ}$

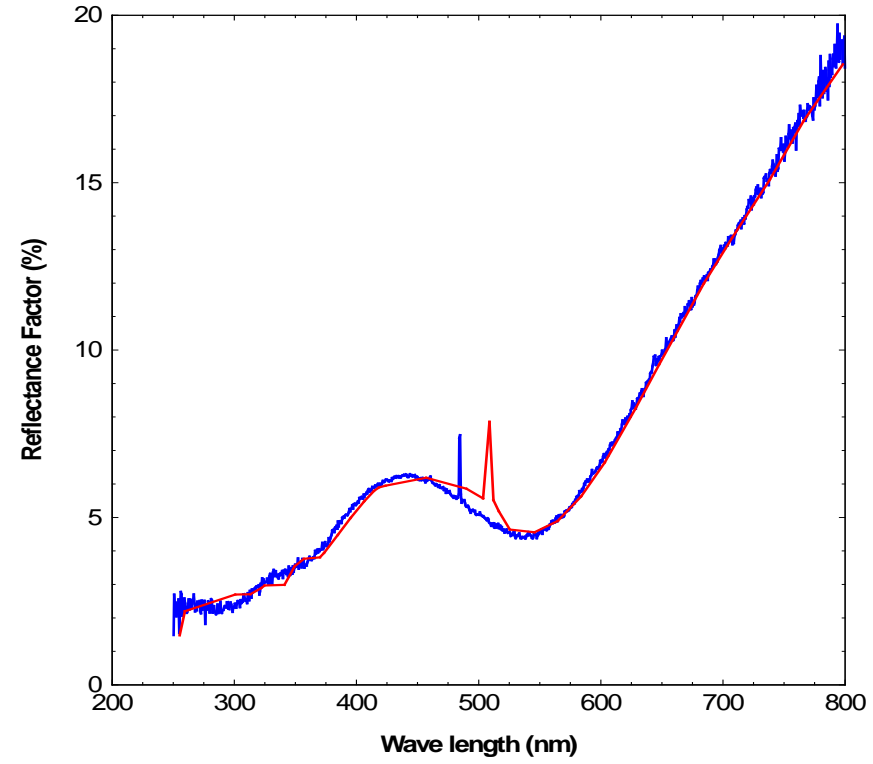

Fig. (5): Junonia reflectance spectrum measured and simulated at an incident angle

$$
\theta=30^{\circ}
$$

Figure (4) shows the results of the numerical calculations. The main peak was estimated at $510 \mathrm{~nm}$, which is within the range of blue. Figure (5) shows the two comparative results of experiments and calculations. We note 
a difference between the spectrum measured at $483 \mathrm{~nm}$ and that calculated at $512 \mathrm{~nm}$. The difference can be explained by the uncertainty angle $\left(30^{\circ}\right)$ and also, the disorder present in the real structure, responsible for the variation of thickness and refractive indices of the multilayer. This numerical result is in good agreement with the results in measurement. This confirms that the multilayer observed with the SEM is responsible for the blue coloration of the blue wings of the butterfly Junonia.

\section{Conclusion}

The results of the spectrophotometer measurements show a main peak at $483 \mathrm{~nm}$. The reflected wavelength deduced by calculating is estimated at $515 \mathrm{~nm}$. The numerical results show a main peak at $512 \mathrm{~nm}$. There is good agreement between the measured results and those of our calculations. The gap between the different measurement methods or numerical calculation is essentially due to uncertainty about the angles, thicknesses and especially the refractive indices of the multilayer. The overall results show that a multilayer is the origin of blue coloration of the wings of the butterfly Junonia. So it is called structural coloration. SEM images allow highlighting the structure which is at the origin of the blue coloration. This structure is thin with about $200 \mathrm{~nm}$. Finally, we can consider the reproduction of the visual rendering of the butterfly Junonia by deposition techniques to achieve an artificial multilayer.

\section{Acknowledgements}

The authors want to thank the "Cooperation Universitaire de Developpement CUD” Projet Intérêts Créditeur PIC 2012-2015, between the Solid Physics Laboratory of the University of Namur,Belgium. URF-SEA, University of Ouaga I. Pr Joseph Ki ZERBO, the Energy Department ED, and the National Scientific Research Technological Centre CNRST of Burkina Faso.

\section{References:}

Biro, L. P; Kertész, K., Vértesy, Z., Màrk, G. I., Balint, Zs., Lousse, V. and Vigneron, P.J. (2007). Living photonic crystals: butterfly scalesnanostructure and optical properties. Mat. Sci. Eng.

Vukusic, P; and Sambles, J. R. (2003). Photonic structures in biology. (pp. 852-855). Nature Journal. (Doi: 10.1038/nature01941).

Land, M. The physics and biology of animal reflectors. (1972). Prog. Biophys. Mol. Biol. (pp.75).

Nekrutenko, Y. P. (1965). Gynandromorphic effect and the optical nature of hidden wing pattern in gonepteryx rhamni (Lepidoptera, pieridae). Natu.re Journal (pp.417). 
Vigneron, J. P; Colomer, J. F; Rassart, M. A. Ingram L. and Lousse, V. (2006). Structural origin of the colored reflections from the black-billed magpie feathers. Phys. Rev. E, 73:021914(pp. 1-7).

Balint, Zs; Vértesy, Z; and Biro, L. P. (2005). Microstructures and nanostructures of high Andean Penaincisalia lycaenid butterfly scales (Lepidoptera: Lycaenidae): descriptions and interpretations. J. Nat. Hist. 39. (pp.2935-2952). (Doi: 10.1080/00222930500140629).

Berthier, S. (2007). Iridescences, the Physical Colors of Insects. SpringerVerlag, Paris, France.

ICI. (1931). International Commission on Illumination (ICI). Cambridge University Press, Proceedings.

Prendry, J; and Kinnon, Mc. (2005). Calculation of photon Dispersion relations. Phys. Rev. Lett. 69. (pp.2772).

Yeh, P. (2005). Optical Wave in layered. (Media Wiley).

Hoo, Y; Jin, W; Shi, C. (2003). Design and Modeling of a photonic crystal fiber Gas Sensor. Appl. Opt. (pp.3509).

Lafait, J; Andraud, C; Berthier, S. (2010). Modeling the vivid white color of the beetle Calothyrza Margaritifera. Mat. Sci. Eng. B-Solid state Matter. Adv Technol.

Kertesz, K; Molnar, G; Vertesy, Z. (2008). Photonic band gap materials in butterfly scales: A possible source of blueprints. Materials Science and Engineering B 149. (pp. 259-265).

Biró, L. P; Vértesy, Z; Márk, G; Lousse, V; and Vigneron, J. P. (2003). Role of photonic- crystal-type sructures in the thermal regulation of a lycaenid butterfly sister species pair. Phys. Rev. E, 67:021907. (pp. 1-7).

Zi, J; Yu, X; Li, Y; Hu, X; Xu, C; Wang, X; Liu, X; and Fu, R.(2003). Coloration strategies in peacock feathers. In Proc. Natl. Acad. Sci., volume 100, (pp.12576), USA.

Potyrailo, R; Giradella, H; Vertiachikh, A. (2007). Morpho Butterfly wing scales demonstrate highly selective vapour response. Nat. Photonics 1, (pp. 123).

Vigneron, J. P; Simonis, P; Aeillo, A.(2010). Reverse Color Sequence in the diffraction of white Light by the wing of the mal butterfly Pierella luna. Phys. Rev. E.

Watanabe, K; Hoshimo, T; Kanda, K; Haruyama, Y; Kaito, T. (2005). Matsui, S. Optical measurement and fabrication from a Morpho-butterflyscale quasi-structure by focused ion beam chemical vapor deposition. B23. (pp.570-574). DOI 10.1116/1.1868697.

Mason C. W. (1927). Structural colors in insects. J. Phys. Chem. 